Check for updates

Cite this: RSC Adv., 2017, 7, 26250

\title{
Investigating resonance energy transfer from protein molecules to van der Waals nanosheets
}

Received 25th February 2017

Accepted 5th May 2017

DOI: 10.1039/c7ra02376f

rsc.li/rsc-advances

\author{
Arun Singh Patel, ${ }^{a}$ Praveen Mishra, ${ }^{a}$ Pawan K. Kanaujia, ${ }^{\text {b }}$ Syed Shariq Husain, ${ }^{a}$ G. Vijaya \\ Prakash $^{\mathrm{b}}$ and Anirban Chakraborti (D) *a
}

The resonance energy transfer (RET) from tryptophan present in bovine serum albumin (BSA) to two dimensional (2D) nanomaterials has been reported. In these bio-nano systems, BSA molecules act as efficient donors while 2D van der Waals nanosheets of graphene, molybdenum disulfide $\left(\mathrm{MoS}_{2}\right)$ and tungsten disulfide $\left(\mathrm{WS}_{2}\right)$ act as acceptors. The fluorescence emission from tryptophan arises at $350 \mathrm{~nm}$, in presence of 2D nanosheets, the fluorescence intensity of BSA decreases. The time resolved fluorescence study shows that there is decrease in the fluorescence lifetime of BSA molecules in presence of 2D nanosheets. The decrease in fluorescence lifetime of BSA confirms the energy transfer phenomenon in bio-nano interactions.

\section{Introduction}

In recent years, two dimensional (2D) nanosheets of van der Waals (vdW) materials have been extensively studied due to their intriguing optical, electronic, structural and chemical properties. ${ }^{1-5}$ These materials include a wide range of nanomaterials zero band gap to non-zero band gap. Among various types of vdW nanomaterials, graphene and transition metal dichalcogenides (TMDCs) nanosheets of the $\mathrm{MX}_{2}$ type $(\mathrm{M}=\mathrm{Mo}, \mathrm{W} ; \mathrm{X}=\mathrm{S}, \mathrm{Se}, \mathrm{Te})$, are the most studied materials. ${ }^{6-9}$ These materials have the advantage over other nanomaterials due to their high stability, high surface to volume ratio and a number of layer dependent electronic and optical properties. ${ }^{10-12}$ Graphene consists of carbon atoms, arranged in a regular atomic-scale chicken wire (hexagonal) pattern. The emergence of graphene helped further the exploration of other two dimensional nanomaterials like, 2D sheets of TMDCs. ${ }^{13,14}$ Among various TMDCs nanosheets, $\mathrm{MoS}_{2}$ and $\mathrm{WS}_{2}$ have been lately used in various optoelectronic devices. In bulk form, these TMDCs are found to be indirect band gap semiconductors, while for single layers, they are direct band gap semiconductors with different band gap energies, e.g., $\mathrm{MoS}_{2}$ has $1.2 \mathrm{eV}$ indirect band gap in bulk form, while for single layer it has direct band gap of $1.8 \mathrm{eV}$, and similarly, $\mathrm{WS}_{2}$ has $1.4 \mathrm{eV}$ indirect band gap in bulk form, while in case of single layer it is a $2.1 \mathrm{eV}$ direct band gap semiconductor. ${ }^{15-19}$ Recently, graphene as well as TMDC nanosheets have been used in resonance energy transfer (RET) phenomenon as acceptors of energy/charge. ${ }^{20-25}$ The RET phenomenon is observed in various physical, chemical and

${ }^{a}$ School of Computational \& Integrative Sciences, Jawaharlal Nehru University, New Delhi-110067, India. E-mail: anirban@jnu.ac.in; Tel: +91-11-26704624

${ }^{b}$ Nanophotonics Laboratory, Department of Physics, Indian Institute of Technology Delhi, New Delhi-110016, India biological systems. ${ }^{26,27}$ In order to observe RET, one material acts as a donor and the other material as an acceptor of energy. Very recently, Sampat et al. has studied RET from quantum dots to $\mathrm{MoS}_{2}$ nanosheets, Goodfellow et al. used CdSe/CdS QDs as donors and $\mathrm{WS}_{2}$ nanosheets as acceptor in RET. ${ }^{20,24}$ Similarly, Swathi et al. used a fluorescent dye as a donor and graphene as an acceptor for resonance energy transfer. ${ }^{28}$ There are fewer studies on RET in biomolecules like protein and nanomaterials. Sen et al. have investigated RET in human serum albumin (HSA) protein molecules and gold nanoparticles. ${ }^{29}$ Recently, Kuchlyan et al. have studied the interaction of BSA molecules with graphene oxide nanosheets. ${ }^{30}$ Prior to application of nanomaterials in biological systems, testing the biocompatibility of the nanomaterials is essential. In this regard, the interaction of two dimensional nanosheets with biomolecules needs to be explored before their use in diagnosis and biomedical applications. The present study is important in the context of application of two dimensional nanosheets in bio-related fields, as for the first time here, we have investigated the bio-nano interaction in 2D nanosheets and bovine serum albumin molecules by means of resonance energy transfer.

\section{Materials and methods}

\subsection{Materials}

Bovine serum albumin, graphite powder, molybdenum disulfide $\left(\mathrm{MoS}_{2}\right)$, tungsten disulfide $\left(\mathrm{WS}_{2}\right)$ were obtained from Sigma-Aldrich and used without further purification. In all the experiments, ultrapure double distilled water was used.

\subsection{Synthesis of van der Waals nanosheets}

In this study, the van der Waals (vdW) nanosheets were synthesized by using the liquid exfoliation method. In a typical 
synthesis procedure, $50 \mathrm{mg}$ of $\mathrm{vdW}$ powder was dispersed in 10 $\mathrm{mL}$ of aqueous solution containing $10 \mathrm{mg}$ BSA powder. Further the solution was ultrasonicated for $20 \mathrm{~h}$ using $25 \mathrm{~W}$ ultrasonication bath. After ultrasonication, the color of the solution changed, e.g., for $\mathrm{MoS}_{2}$ from black to green, which confirmed the formation of nanosheets from bulk powder. Similarly, the color of $\mathrm{WS}_{2}$ changed to brown, while that of graphene remained black. The supernatant was then collected and redispersed in water to prepare diluted stock solutions $(0.05 \mathrm{mg}$ $\mathrm{mL}^{-1}$ ) of $\mathrm{vdW}$ nanosheets. The schematic diagram of the chemical exfoliation process is shown in Fig. 1.

\subsection{Characterization}

The solutions were drop casted on silicon substrates and dried at room temperature for Raman spectroscopic studies. These nanosheets were characterized by transmission electron microscopy (TEM), Raman spectroscopy and absorption spectroscopy. The Raman spectra of graphene, $\mathrm{MoS}_{2}$ and $\mathrm{WS}_{2}$ nanosheets were recorded by Renishaw inVia confocal Raman spectrometer using $532 \mathrm{~nm}$ excitation source. For TEM study, the aqueous dispersions of the nanosheets were put on carbon coated copper grids and dried at room temperature. The TEM images of these nanosheets were recorded using JEOL-2010 TEM setup, which was operated at an accelerating voltage of $200 \mathrm{kV}$. The elemental analysis of the fluorescent protein was done using energy dispersive X-ray (EDX) measurement, which was carried out on ZEISS EVO40-scanning electron microscopy (SEM) set up with energy $15 \mathrm{keV}$. The absorption spectra of these nanosheets were recorded using Shimadzu (UV-2450) UVVis spectrophotometer. In order to study RET in BSA and the van der Waals nanosheets, different quantities of nanosheets were mixed with a fixed quantity of BSA containing aqueous solution. All samples were prepared such that the amount of BSA molecules remains fixed $(0.5 \mathrm{mg}$ in $3.5 \mathrm{~mL}$ solution) in all sets, while only the quantities of the nanosheets were varied in different samples. All samples were incubated for $24 \mathrm{~h}$ to achieve equilibrium. The steady state fluorescence emissions of BSA, in absence and presence of nanosheets, were recorded using fluorimeter with excitation wavelength of $280 \mathrm{~nm}$. The

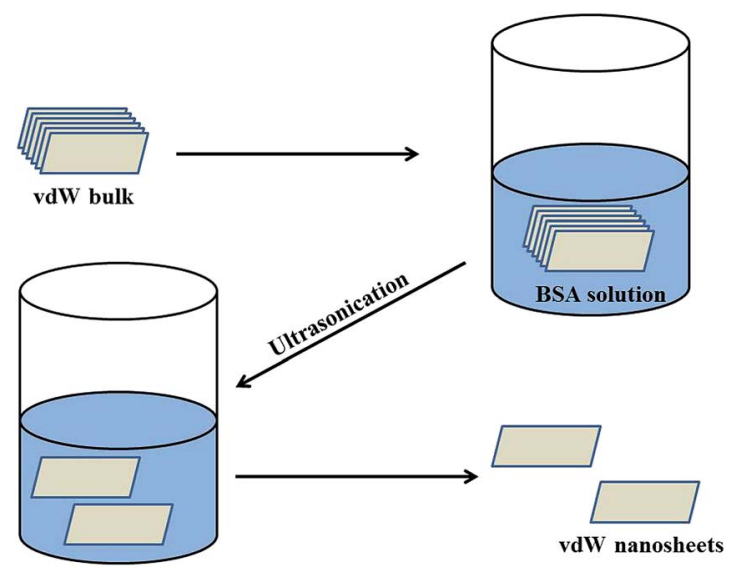

Fig. 1 Schematic diagram for synthesis of van der Waals nanosheets. time resolved fluorescence study was performed using time correlated single photon counting (TCSPC) setup (FL920, Edinburgh Instruments, UK) with $284 \mathrm{~nm}$ light emitting diode as the excitation source.

\section{Results and discussion}

\subsection{Raman study}

The Raman spectra of the van der Waals nanosheets are shown in Fig. 2. Fig. 2a shows the Raman spectrum of graphene nanosheet. In case of graphene, three distinct Raman peaks are observed: 1350,1582 and $2700 \mathrm{~cm}^{-1}$; these peaks are known as $\mathrm{D}, \mathrm{G}$ and $2 \mathrm{D}$ peaks, respectively. ${ }^{31} \mathrm{D}$ and $\mathrm{G}$ peaks originate due to the breathing modes of $\mathrm{sp}^{2}$ carbon atoms in the ring and stretching of carbon-carbon bonds respectively. The Raman spectrum of $\mathrm{MoS}_{2}$ nanosheet is shown in Fig. 2b. There are two distinct Raman peaks: one at $385 \mathrm{~cm}^{-1}$ and the other at 405 $\mathrm{cm}^{-1}$. These peaks arise due to in-plane and out-of-plane vibrations of S-Mo-S atoms, and are known as $\mathrm{E}_{2 \mathrm{~g}}{ }^{1}$ and $\mathrm{A}_{1 \mathrm{~g}}$ vibrational modes, respectively. ${ }^{32}$ In case of $\mathrm{WS}_{2}$ nanosheets, $\mathrm{E}_{2 \mathrm{~g}}{ }^{1}$ and $\mathrm{A}_{1 \mathrm{~g}}$ Raman peaks are observed at $351 \mathrm{~cm}^{-1}$ and 420 $\mathrm{cm}^{-1}$, respectively.

\subsection{TEM analysis}

The morphologies of two dimensional vdW nanosheets of graphene, $\mathrm{MoS}_{2}$ and $\mathrm{WS}_{2}$, were analyzed by transmission electron microscopy (TEM). Fig. 3a shows the TEM image of graphene nanosheets. The selective area electron diffraction (SAED) of graphene is shown in Fig. 3b. Similarly, the TEM images of $\mathrm{MoS}_{2}$ and $\mathrm{WS}_{2}$ are shown in Fig. $3 \mathrm{c}$ and e, respectively, and the Fig. $3 \mathrm{~d}$ and $\mathrm{f}$ show the selective area electron diffraction (SAED) patterns of $\mathrm{MoS}_{2}$ and $\mathrm{WS}_{2}$ nanosheets, respectively.

\subsection{EDX analysis}

The elemental analysis of the fluorophore (BSA protein) molecules was done by using EDX study. The EDX spectrum of BSA is shown in Fig. 4. The spectrum consists of peaks corresponding to different elements present in BSA molecules. In EDX spectrum the peaks corresponding to carbon, nitrogen, oxygen, sodium and sulfur are observed at 0.277, 0.392, 0.525, 1.041 and $2.307 \mathrm{keV}$, respectively. Additional peak corresponding to silicon is observed at $1.739 \mathrm{keV}$, which originates due to the silicon substrate.

\subsection{UV-visible absorption}

The optical absorbance spectra of vdW nanosheets were recorded by the absorption spectrophotometer, and are shown in Fig. 5 .

The absorption spectrum of graphene shows an absorption peak around $280 \mathrm{~nm}$. This absorption peak arises due to $\pi$ conjugation, which is partially restored within the graphene sheets. In case of $\mathrm{MoS}_{2}$, two absorption peaks are observed; one at $620 \mathrm{~nm}$ and other at $670 \mathrm{~nm}$. These peaks are the resultant of transitions between valence band and conduction band of $\mathrm{MoS}_{2}$. The absorption peaks observed at $620 \mathrm{~nm}$ and $670 \mathrm{~nm}$ are assigned as B and A exciton peaks, respectively. Similarly, for 

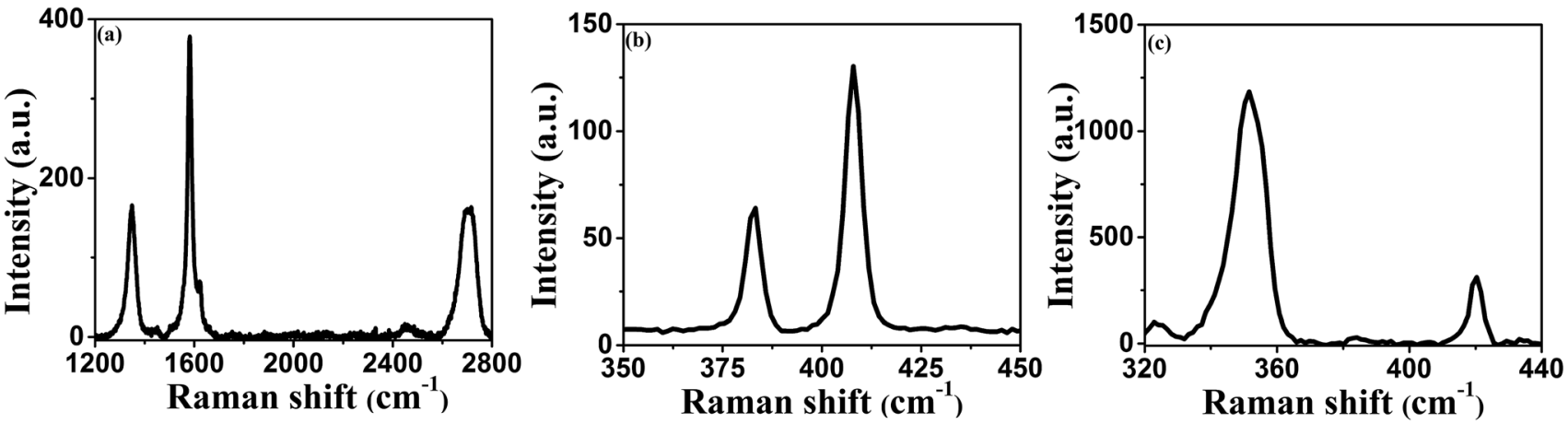

Fig. 2 Raman spectra of van der Waals nanosheets of (a) graphene, (b) $\mathrm{MoS}_{2}$, and (c) $\mathrm{WS}_{2}$.

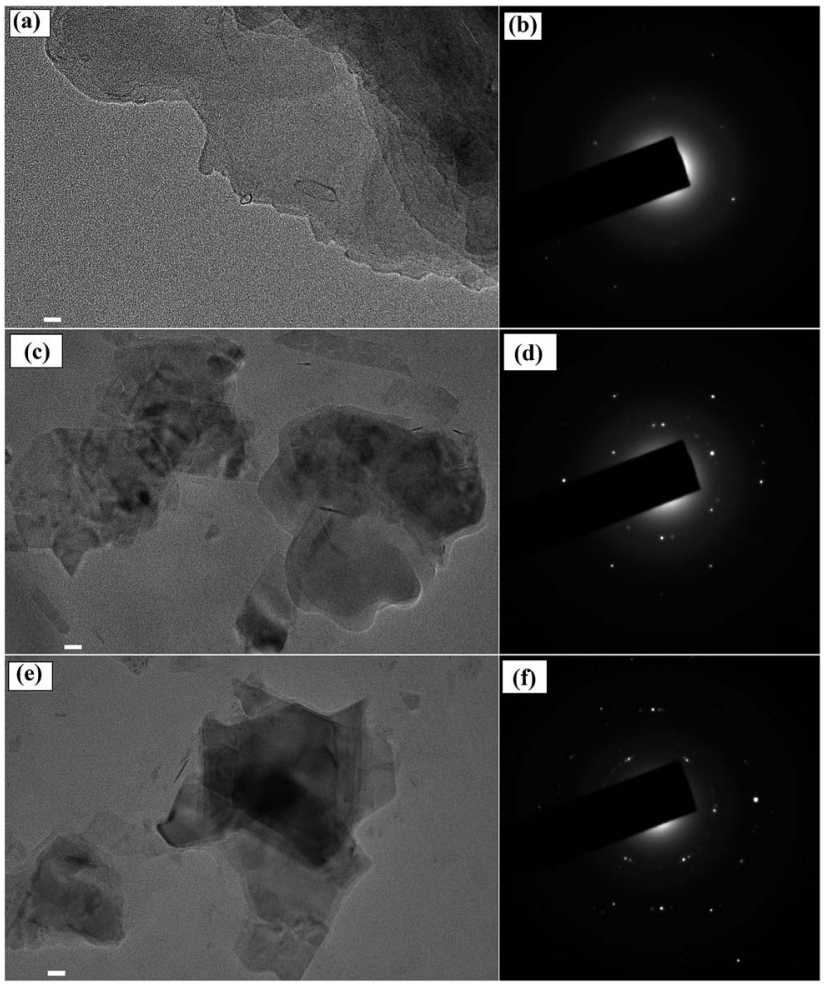

Fig. 3 TEM images and SAED patterns of (a) and (b) graphene, (c) and (d) $M_{0}$, (e) and (f) $W_{2}$ nanosheets, respectively. Scale bar equals to $20 \mathrm{~nm}$.

$\mathrm{WS}_{2}$ nanosheets the absorption peaks are observed at $530 \mathrm{~nm}$ and $630 \mathrm{~nm}$. The absorption peaks are similar to the previous ones reported..$^{33}$

\subsection{Fluorescence study}

The interaction of these nanosheets with BSA molecules was studied by using fluorescence spectroscopic techniques. The steady state fluorescence spectra of BSA molecules in absence and presence of $2 \mathrm{D}$ nanosheets are recorded by fluorimeter using $280 \mathrm{~nm}$ excitation wavelength, and emission was recorded in wavelength range of $290-500 \mathrm{~nm}$. The steady state fluorescence spectra of BSA molecules in presence of different concentration of 2D nanosheets are shown in Fig. 6. BSA shows

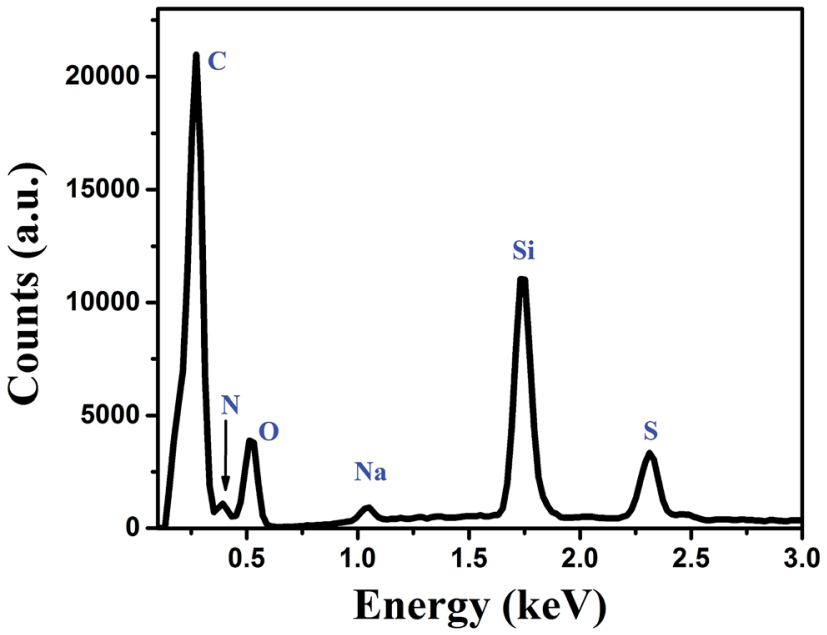

Fig. 4 Energy dispersive $\mathrm{X}$-ray spectrum of the fluorescent BSA molecules.

fluorescence emission at $350 \mathrm{~nm}$, which arises due to tryptophan present in BSA molecules. ${ }^{34}$ In Fig. 6a, it is found that with increase in the concentration of graphene nanosheets, the fluorescence $(\mathrm{Fl})$ intensity of BSA decreases. The decrease in Fl intensity confirms quenching due to graphene nanosheets. In Fig. $6 \mathrm{~b}$ fluorescence quenching of BSA molecules in presence of $\mathrm{MoS}_{2}$ nanosheets is shown. Similarly, for $\mathrm{WS}_{2}$ nanosheets, the quenching of BSA fluorescence is shown in Fig. 6c. The fluorescence quenching of BSA molecules is confirmed, but in order to ascertain the quenching nature (static or dynamic), time resolved fluorescence study was done using time correlated single photon counting technique. It is observed that the fluorescence lifetime of BSA decays, in absence and presence of different concentrations of vdW nanosheets. The fluorescence lifetime decay spectra of BSA, in the absence and presence of $2 \mathrm{D}$ nanosheets, are shown in Fig. 7. Fig. 7a shows the Fl lifetime decay curve of BSA molecules in presence of graphene nanosheets. It is observed that with increase in the quantity of graphene, the fluorescence decay curves are steeper in comparison to pure BSA. There is a systematic change in the decay curves with increase in the quantity of graphene nanosheets. Similarly, Fig. $7 \mathrm{~b}$ and $\mathrm{c}$ show the fluorescence lifetime decays of BSA, in 


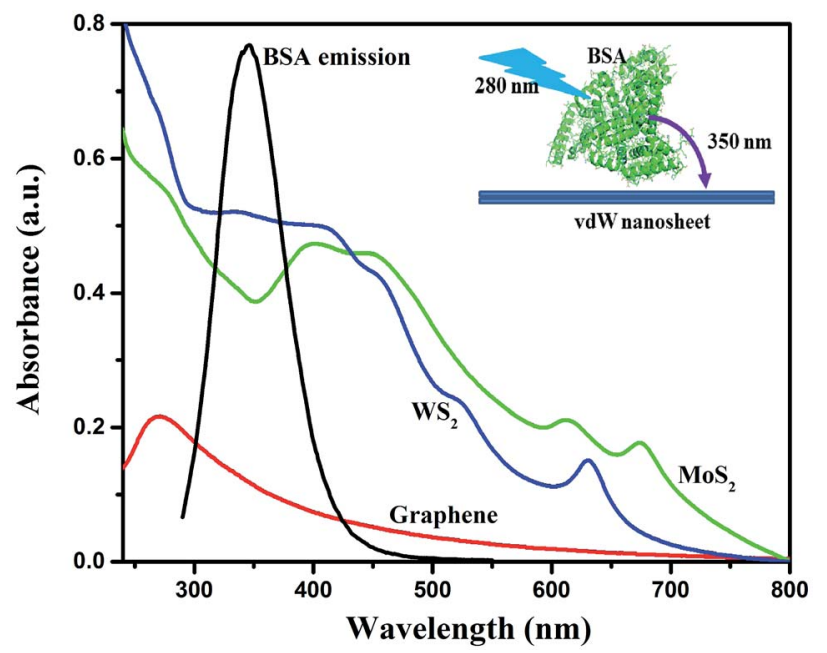

Fig. 5 Absorption spectra of graphene, $\mathrm{MoS}_{2}$ nanosheets, and $\mathrm{WS}_{2}$ nanosheets, along with the emission spectrum of BSA. Inset: schematic representation of energy transfer between BSA and the van der Waals nanosheets.

presence of $\mathrm{MoS}_{2}$ and $\mathrm{WS}_{2}$ nanosheets, respectively. These decay curves were fitted with exponential decaying functions of following form: ${ }^{35}$

$$
I(t)=\sum_{j=1}^{m} \alpha_{j} \exp \left(-t / \tau_{j}\right),
$$

where $m$ is the number of discrete decay components, $\tau_{j}$ are the decay times, and $\alpha_{j}$ represents the weighing parameter associated with the $j^{\text {th }}$ decay. The decay curve of BSA in absence of nanosheets is fitted with bi-exponential $(m=2)$ decaying function, while in presence of nanosheets the curves are fitted with triple-exponential decaying function (fitted curves not shown). The average fluorescence lifetimes of BSA in absence, as well as in presence of nanosheets, were calculated using following equation: ${ }^{36}$

$$
\langle\tau\rangle=\frac{\sum_{j=1}^{m} \alpha_{j} \tau_{j}}{\sum_{j=1}^{m} \alpha_{j}} .
$$

The average fluorescence lifetime of pure BSA was found to be $6.41 \mathrm{~ns}$. In presence of different quantities of graphene, the $\mathrm{Fl}$ lifetime decreases - for $0.1,0.3,0.5$ and $0.7 \mathrm{~mL}$ of graphene solutions, the Fl lifetimes are found to be 6.08, 5.89, 5.66 and $5.55 \mathrm{~ns}$, respectively. Thus, there is a systematic decrease in the Fl lifetimes of BSA molecules in presence of different quantities of graphene. Similarly, for $\mathrm{MoS}_{2}$ nanosheets, the respective $\mathrm{Fl}$ lifetimes are found to be $6.39,6.22,5.70$ and $5.62 \mathrm{~ns}$ in presence of $0.1,0.3,0.5$ and $0.7 \mathrm{~mL}$ of $\mathrm{MoS}_{2}$ nanosheets. For $\mathrm{WS}_{2}$ the values are $6.26,5.93,5.61,5.44 \mathrm{~ns}$ with $0.1,0.3,0.5$ and $0.7 \mathrm{~mL}$ of $\mathrm{WS}_{2}$ solutions, respectively. In all cases, with increase in the quantity of vdW nanosheets, there is a corresponding decrease in the Fl lifetime of BSA molecules. In presence of $2 \mathrm{D}$ nanosheets, the fluorescence intensity as well as the fluorescence lifetime of BSA molecules are found to decrease. In Fig. 8, variation of $\mathrm{Fl}$ intensity and Fl lifetime of BSA with different quantities of 2D nanosheets are shown. The decrease in lifetime indicates that there is some energy transfer from the BSA molecules to $2 \mathrm{D}$ nanosheets. In Fig. 5 , it is shown that the emission spectrum of BSA overlaps with the absorption spectra of vdW nanosheets. This results in transfer of some energy from BSA molecules to the nanosheets. In the process of energy transfer, the fluorophores show quenching in the Fl intensity, as well as Fl lifetimes. Using Fl lifetime data, the energy transfer efficiency from BSA molecules to 2D nanosheets has been calculated using following equation:

$$
E=1-\frac{\tau_{\mathrm{DA}}}{\tau_{\mathrm{D}}} .
$$

Here, $\tau_{\mathrm{D}}$ and $\tau_{\mathrm{DA}}$ are fluorescence lifetimes of BSA, in absence and presence of $2 \mathrm{D}$ nanosheets (acceptors), respectively. It is found that with increase in the concentration of acceptors the energy transfer efficiency increases. The energy transfer efficiencies, in case of graphene are found to be 5, 8, 12 and 13\% for $0.1,0.3,0.5$ and $0.7 \mathrm{~mL}$ of graphene solutions, respectively. For $\mathrm{MoS}_{2}$ the efficiencies are found to be $0.5,3,11$ and $12 \%$ with $0.1,0.3,0.5$, and $0.7 \mathrm{~mL}$ of $\mathrm{MoS}_{2}$ solutions, respectively. Finally, in the case of $\mathrm{WS}_{2}$ nanosheets, the efficiencies were 2, 7, 12 and $15 \%$ for $0.1,0.3,0.5$ and $0.7 \mathrm{~mL}$ of the solutions, respectively.

The energy transfer efficiency was also calculated using steady state fluorescence emission data of BSA, in absence as
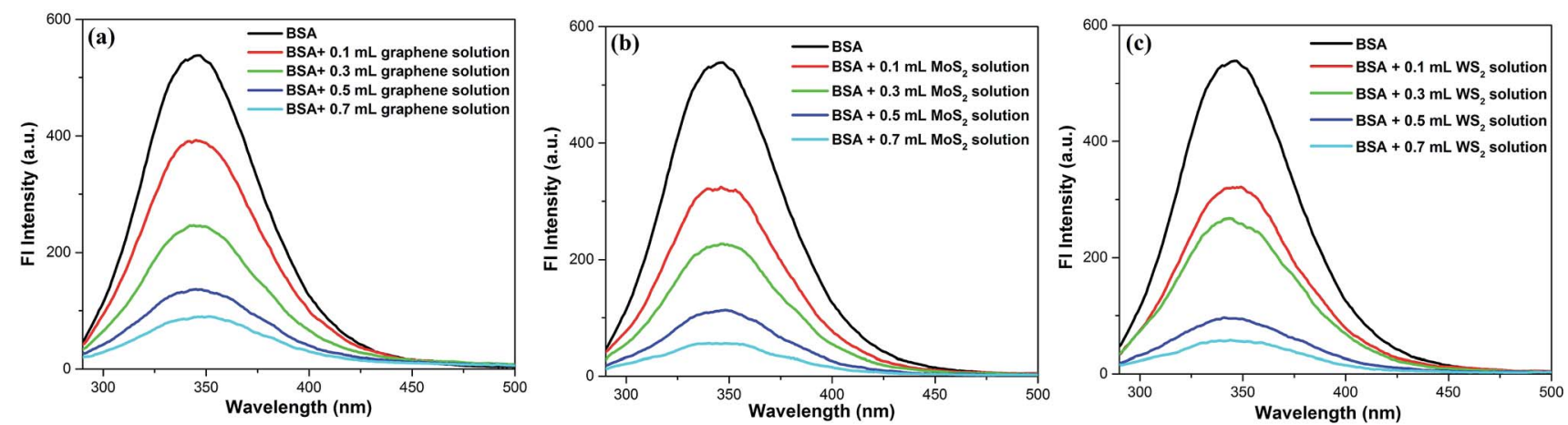

Fig. 6 Fluorescence spectra of BSA in absence and presence of different quantities of (a) graphene, (b) MoS $S_{2}$ and (c) WS $S_{2}$ nanosheets, respectively. 

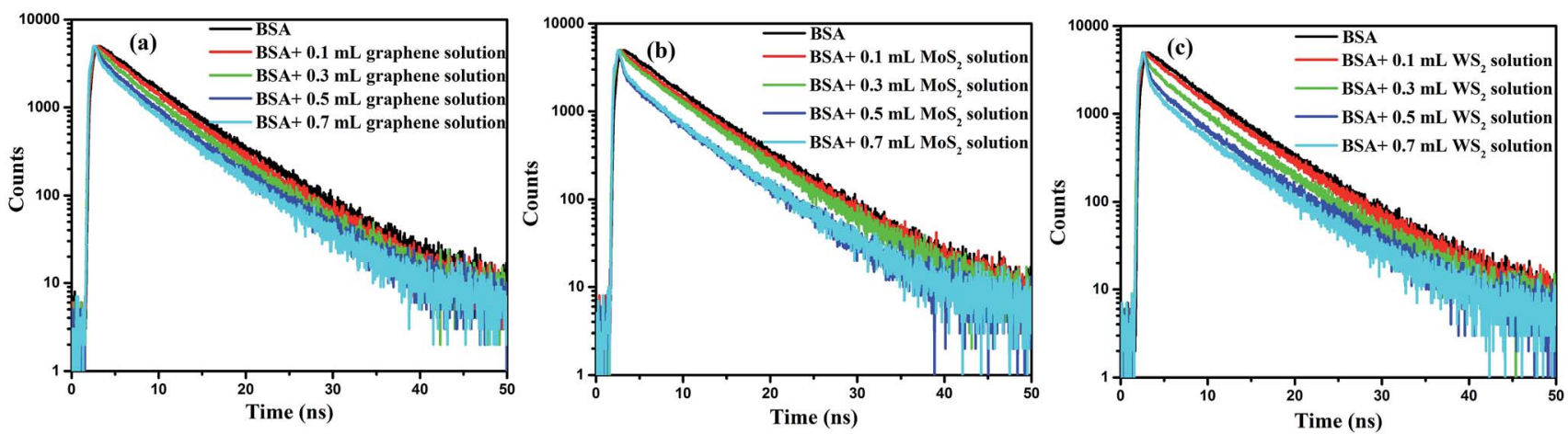

Fig. 7 Fluorescence lifetime decay curves of BSA, in the absence and in the presence of different quantities of (a) graphene, (b) MoS 2 , and (c) WS 2 nanosheets, respectively.
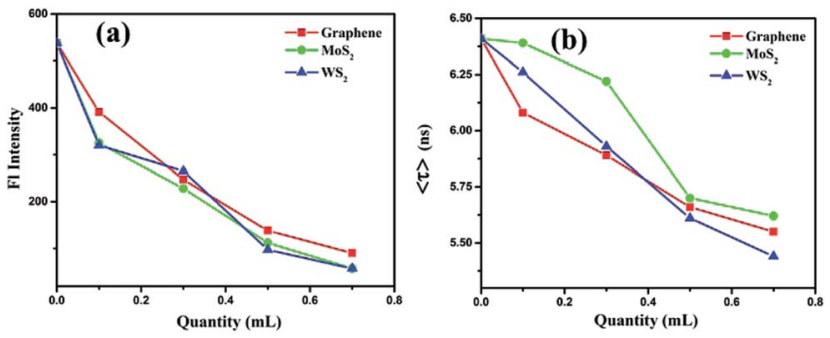

Fig. 8 Variation of: (a) fluorescence intensity and (b) fluorescence lifetime of BSA molecules, in presence of different quantities of 2D nanosheets.

well as presence of different vdWs nanosheets, using following equation:

$$
E=1-\frac{F_{\mathrm{DA}}}{F_{\mathrm{D}}},
$$

where $F_{\mathrm{D}}$ is fluorescence intensity of BSA in absence of acceptors, while $F_{\mathrm{DA}}$ is intensity in presence of acceptors. The energy transfer efficiencies were found to be $27,54,74$ and $83 \%$ for 0.1 , $0.3,0.5$ and $0.7 \mathrm{~mL}$ of graphene solutions, respectively. In case of $\mathrm{MoS}_{2}$, the efficiencies were 40, 58,79 and $89 \%$ for $0.1,0.3,0.5$ and $0.7 \mathrm{~mL}$ of $\mathrm{MoS}_{2}$ solutions, respectively. Similarly, for $\mathrm{WS}_{2}$, the efficiencies were found to be $40,51,82$ and $89 \%$ for $0.1,0.3$, 0.5 and $0.7 \mathrm{~mL}$ of the solutions, respectively.

There is a difference in the energy transfer efficiency values for Fl intensity data and Fl lifetime data, which indicates possibilities of two kinds of Fl quenching mechanisms, viz., static and dynamic. The decrease in fluorescence lifetimes normally corresponds to the dynamic nature of Fl quenching, and possibility of the resonance energy transfer from BSA to $2 \mathrm{D}$ nanosheets, while quicker decay in the Fl intensity confirms the static nature of quenching. In Fig. 5, it has been shown that the fluorescence emission of BSA molecules overlaps with some fraction of the absorption spectrum of the 2D nanosheets. The overlapping fluorescence of BSA and absorption of nanosheets causes transfer of energy from BSA molecules to the nanosheets. When the BSA molecules are excited by $280 \mathrm{~nm}$ excitation light, they emit at higher wavelength of $350 \mathrm{~nm}$. In presence of nanosheets, some fraction of this emission gets absorbed by the nanosheets, which results in decrease in fluorescence intensity, as well as the fluorescence lifetimes of the BSA molecules. At the same time, the BSA molecules are attached to the nanosheets by van der Waals interaction and form BSA-nanosheets complexes, which is not fluorescent. Therefore, there is a steeper decrease in the fluorescence intensity of BSA molecules, in presence of nanosheets, as compared to decrease in the Fl lifetimes. The quenching of Fl intensity as well as Fl lifetimes can play important role for detection of foreign molecules/ions. The addition of probe molecules/ions can affect the fluorescence intensity or fluorescence lifetimes, depending upon the nature of fluorescence intensity the BSATMDCs composites can be used as fluorescence turn ON/OFF sensor.

\section{Conclusions}

In summary, we have studied the resonance energy transfer between BSA molecules and two dimensional nanosheets of graphene, $\mathrm{MoS}_{2}$ and $\mathrm{WS}_{2}$. This study confirms that these nanosheets can be used as efficient acceptors for energy transfer studied in biomolecules, which can be further explored to study the effect of 2D nanomaterials on biomolecules, prior to application of these nanomaterials in biological applications. The resonance energy transfer can be explored further to detect molecules/ions which have effect on the RET efficiency. This study opens a new set of bio-nano composites, which can be used as sensor of molecules/ions.

\section{Acknowledgements}

$\mathrm{AC}$ and ASP acknowledge financial support from grant number BT/BI/03/004/2003(C) of Government of India, Ministry of Science and Technology, Department of Biotechnology, Bioinformatics division, and the DST-PURSE grant given to Jawaharlal Nehru University by the Department of Science and Technology, Government of India. Authors thank the AIRF, JNU for TEM, EDX, TRFS characterizations and the FIST (DST, Govt. of India) UFO scheme of IIT Delhi for Raman/PL facility. 


\section{References}

1 W. Chen, E. J. Santos, W. Zhu, E. Kaxiras and Z. Zhang, Nano Lett., 2013, 13, 509-514.

2 Y. Shi, J.-K. Huang, L. Jin, Y.-T. Hsu, S. F. Yu, L.-J. Li and H. Y. Yang, Sci. Rep., 2013, 3, 1839.

3 K. F. Mak, K. He, C. Lee, G. H. Lee, J. Hone, T. F. Heinz and J. Shan, Nat. Mater., 2013, 12, 207-211.

4 L. Falkovsky and S. Pershoguba, Phys. Rev. B: Condens. Matter Mater. Phys., 2007, 76, 153410.

5 Y. Ding, Y. Wang, J. Ni, L. Shi, S. Shi and W. Tang, Phys. B, 2011, 406, 2254-2260.

6 A. K. Geim and K. S. Novoselov, Nat. Mater., 2007, 6, 183-191.

7 B. Radisavljevic, A. Radenovic, J. Brivio, I. V. Giacometti and A. Kis, Nat. Nanotechnol., 2011, 6, 147-150.

8 H. Ramakrishna Matte, A. Gomathi, A. K. Manna, D. J. Late, R. Datta, S. K. Pati and C. Rao, Angew. Chem., 2010, 122, 4153-4156.

9 Q. H. Wang, K. Kalantar-Zadeh, A. Kis, J. N. Coleman and M. S. Strano, Nat. Nanotechnol., 2012, 7, 699-712.

10 H. Hibino, H. Kageshima, M. Kotsugi, F. Maeda, F.-Z. Guo and Y. Watanabe, Phys. Rev. B: Condens. Matter Mater. Phys., 2009, 79, 125437.

11 C. Lee, H. Yan, L. E. Brus, T. F. Heinz, J. Hone and S. Ryu, ACS Nano, 2010, 4, 2695-2700.

12 W. Zhao, R. M. Ribeiro, M. Toh, A. Carvalho, C. Kloc, A. Castro Neto and G. Eda, Nano Lett., 2013, 13, 5627-5634.

13 M. Chhowalla, H. S. Shin, G. Eda, L.-J. Li, K. P. Loh and H. Zhang, Nat. Chem., 2013, 5, 263-275.

14 C. Zhang, Y. Chen, J.-K. Huang, X. Wu, L.-J. Li, W. Yao, J. Tersoff and C.-K. Shih, Nat. Commun., 2016, 7, 10349.

15 A. Chakraborti, A. S. Patel, P. K. Kanaujia, P. Nath, G. V. Prakash and D. Sanyal, Phys. Lett. A, 2016, 380, 40574061.

16 K. F. Mak, C. Lee, J. Hone, J. Shan and T. F. Heinz, Phys. Rev. Lett., 2010, 105, 136805.

17 R. Ganatra and Q. Zhang, ACS Nano, 2014, 8, 4074-4099.

18 J. Shakya, A. S. Patel, F. Singh and T. Mohanty, Appl. Phys. Lett., 2016, 108, 013103.
19 A. Berkdemir, H. R. Gutiérrez, A. R. Botello-Méndez, N. Perea-López, A. L. Elías, C.-I. Chia, B. Wang, V. H. Crespi, F. López-Urías, J.-C. Charlier and A. Berkdemir, Sci. Rep., 2013, 3, 1755.

20 S. Sampat, T. Guo, K. Zhang, J. A. Robinson, Y. Ghosh, K. P. Acharya, H. Htoon, J. A. Hollingsworth, Y. N. Gartstein and A. V. Malko, ACS Photonics, 2016, 3, 708-715.

21 H. Zang, P. K. Routh, Y. Huang, J.-S. Chen, E. Sutter, P. Sutter and M. Cotlet, ACS Nano, 2016, 10, 4790-4796.

22 D. Kufer, T. Lasanta, M. Bernechea, F. Koppens and G. Konstantatos, ACS Photonics, 2016, 3, 1324-1330.

23 D. Kufer, I. Nikitskiy, T. Lasanta, G. Navickaite, F. H. Koppens and G. Konstantatos, Adv. Mater., 2015, 27, 176-180.

24 K. M. Goodfellow, C. Chakraborty, K. Sowers, P. Waduge, M. Wanunu, T. Krauss, K. Driscoll and A. N. Vamivakas, Appl. Phys. Lett., 2016, 108, 021101.

25 F. Prins, A. J. Goodman and W. A. Tisdale, Nano Lett., 2014, 14, 6087-6091.

26 P. R. Selvin, Nat. Struct. Biol., 2000, 7, 730-734.

27 K. Shankar, X. Feng and C. A. Grimes, ACS Nano, 2009, 3, 788-794.

28 R. Swathi and K. Sebastian, J. Chem. Phys., 2009, 130, 086101. 29 T. Sen, S. Mandal, S. Haldar, K. Chattopadhyay and A. Patra, J. Phys. Chem. C, 2011, 115, 24037-24044.

30 J. Kuchlyan, N. Kundu, D. Banik, A. Roy and N. Sarkar, Langmuir, 2015, 31, 13793-13801.

31 W. Zhao, M. Fang, F. Wu, H. Wu, L. Wang and G. Chen, J. Mater. Chem., 2010, 20, 5817-5819.

32 L. Yang, X. Cui, J. Zhang, K. Wang, M. Shen, S. Zeng, S. A. Dayeh, L. Feng and B. Xiang, Sci. Rep., 2014, 4, 5649.

33 T. P. Nguyen, W. Sohn, J. H. Oh, H. W. Jang and S. Y. Kim, J. Phys. Chem. C, 2016, 120, 10078-10085.

34 N. Tayeh, T. Rungassamy and J. R. Albani, J. Pharm. Biomed. Anal., 2009, 50, 107-116.

35 A. S. Patel, H. Sahoo and T. Mohanty, Appl. Phys. Lett., 2014, 105, 063112.

36 A. S. Patel, H. Sahoo and T. Mohanty, J. Fluoresc., 2016, 26, 1849-1855. 